\title{
O Que Já Aprendemos?: Educação Médica, Vulnerabilidades e Responsabilidade Social em Tempo de Pandemia
}

\author{
Lessons Learned?: Medical Education, Vulnerabilities and Social Accountability During the Pandemic \\ Gustavo Antonio Raimondi' ${ }^{(}$, Francis Solange Vieira Tourinho"()
}

\section{RESUMO}

Introdução: Com a pandemia da Covid-19 houve um recrudescimento da vulnerabilidade, do empobrecimento e do desemprego. Com base no pressuposto de que uma escola médica responsável e comprometida precisa estar atenta às necessidades básicas da sociedade para que os processos formativos possam atender a elas, torna-se necessário refletir sobre a educação médica em tempos de pandemia.

Objetivo: O presente ensaio buscará refletir criticamente sobre "o que já aprendemos" em relação à educação médica no contexto atual de pandemia da Covid-19.

Desenvolvimento: Em 2010, a revista Lancet publicou um manuscrito intitulado "Health professionals for a new century: transforming education to strengthen health systems in an interdependent world", o qual realiza uma análise histórica e socioepidemiológica sobre a formação em saúde, a fim de refletir sobre as características necessárias para uma formação profissional voltada para o século XXI. Diante disso e dos 20 anos vividos em um novo século, questionamo-nos: como estamos formando profissionais para o século XXI? Estamos reproduzindo padrões tecnicistas com uma "nova roupagem" ou promovendo a responsabilidade social ao longo do processo formativo? Estamos utilizando metodologias ativas com um enfoque técnico ou ampliando a análise a partir da perspectiva da determinação social do processo saúde-doença proposta pelas DCN? Não é nossa proposta apresentar uma resposta simples, mas fomentar o debate para que possamos pensar juntos(as) quais são os caminhos possíveis para ampliarmos nossa formação e realmente pensarmos em processos formativos voltados para a promoção da equidade no século XXI.

Conclusão: Precisamos advogar em função dos usuários do SUS e também despertar em nossos(as) estudantes o olhar para essa necessidade. As demandas sociais da atualidade também exigem competências atitudinais e relacionais dos profissionais da saúde, o que precisa ser colocado em prática na formação desses profissionais.

Palavras-chave: Educação Médica; Iniquidade Social; Vulnerabilidade em Saúde; Responsabilidade Social; Pandemias.

\begin{abstract}
Introduction: Vulnerability, impoverishment and unemployment have all increased during the Covid-19 pandemic. Considering that a socially responsible and committed Medical School needs to understand that there are basic societal needs on which training processes must be based, it has become necessary to reflect on Medical Education during this period of the Covid-19 pandemic.

Objective: This essay will seek to critically reflect on the lessons learned in relation to Medical Education in the current context of the Covid-19 pandemic.

Development: In 2010, the Lancet magazine published a manuscript entitled "Health professionals for a new century: transforming education to strengthen health systems in an interdependent world", which involved an historical and socio-epidemiological analysis on health training, with the aim of reflecting on the required characteristics for professional training geared toward the 21st century. In view of this and the 20 years elapsed of $a$ new century, we ask the question: how are we training professionals for the 21st century? Are we reproducing technicist standards dressed in "new clothing" or are we promoting social responsibility over the course of the training process? Are we using active methodologies with a technical focus or broadening analysis based on a social deterministic perspective of the health-disease process, proposed by the National Curriculum Guidelines? We do not propose to present a simple answer, but rather to foment the debate so that we can think together about the possible paths to broadening our training and really focus on training processes aimed at promoting equality in the 21st century.
\end{abstract}

Conclusion: We must champion causes on behalf of the SUS users, and also bring this need to our students' attention. Modern society also demands certain attitudes and relational skills of health professionals which need to be put into practice in their training.

Keywords: Medical Education; Social Inequity; Health Vulnerability; Social Responsibility; Pandemics.

'Universidade Federal de Uberlândia, Uberlândia, Minas Gerais, Brasil.

"Universidade Federal de Santa Catarina, Florianópolis, Santa Catarina, Brasil.

Correspondência:

Gustavo Antonio Raimondi.

Universidade Federal de Uberlândia, Avenida Pará, 1720, bloco 2U, sala 8, campus Umuarama, Uberlândia, MG, Brasil. CEP: $38400-902$.

E-mail: gustavo.raimondi@gmail.com

Recebido em 11/08/20; Aceito em 26/08/20. 


\section{INTRODUÇÃO}

Em 2010, o Consenso Global de Responsabilidade Social das Escolas Médicas (Global Consensus for Social Accountability of Medical Schools) ${ }^{1}$ trouxe algumas orientações para a promoção da responsabilidade social no processo de ensino-aprendizagem, que engloba ações relacionadas aos(às) estudantes, docentes, técnicos(as) administrativos(as) e demais funcionários das escolas e comunidade. Com base no pressuposto de que uma escola médica socialmente responsável e comprometida precisa estar atenta às necessidades básicas da sociedade, é imprescindível uma avaliação crítica dos fatos para que os processos formativos possam ir ao encontro dessas exigências ${ }^{1,2 .}$

Dez anos após a publicação do consenso, o planeta vivencia um momento de pandemia da coronavirus disease 2019 (Covid-19), e, desde o início dela, a população mundial tem ouvido a frase "Fique em casa" . Dias, semanas e meses se passaram com um panorama desolador: idosos(as) mais isolados(as), escolas fechadas, necessidade do uso de ensino remoto emergencial, profissionais da saúde evitando o retorno para a própria casa e outros(as) falecendo ainda sem um tratamento adequado ${ }^{4}$. Diante desse cenário, começamos a escutar, como forma de apaziguar as emoções ligadas às incertezas da pandemia, que "estamos todos(as) no mesmo barco". Mas que barco é esse? Estamos em jangadas, barcos isolados ou em um iate ou um cruzeiro? Será que nas questões de saúde estamos todos(as) no mesmo barco?

Para encontrarmos possíveis respostas para essas e outras perguntas, que serão exploradas ao longo do ensaio, precisamos (re)conhecer inicialmente que, no Brasil, 63\% das casas chefiadas por mulheres negras estão abaixo da linha de pobreza. Das mulheres que têm dificuldade de acesso frequente à água, as negras representam 68\% desse número, e $71,5 \%$ dos cadastrados no Cadastro Único para Programas Sociais (Cadúnico) são negros 5 . Quando buscamos dados sobre o índice anos potenciais de vida perdidos (APVP) das principais causas de óbitos entre as pessoas negras, percebemos que a violência está tirando a vida muito mais rápido dos jovens negros ${ }^{6}$. Ademais, a situação de pandemia explicita que a mortalidade materna de mulheres negras em decorrência da Covid-19 é três vezes maior do que a média geral da população7.

A Covid-19 tornou evidente aos olhos da sociedade o que já existia, como as vulnerabilidades ${ }^{8}$, e para o que muitas vezes "se fechavam os olhos"10,11. Além disso, houve um recrudescimento da vulnerabilidade, do empobrecimento e do desemprego durante a pandemia. Além disso, grande parte da população brasileira e mundial vivencia todos os dias um estresse habitacional, superlotação nos lares, problemas de saúde mental e física, questões relacionadas à segurança da comunidade, maior criminalidade, bem como baixos níveis de coesão, confiança e recursos.

Nesse sentido, há um silenciamento ${ }^{8}$ ao qual algumas camadas da sociedade estão submetidas que as impede de ver e enxergar as crianças que moram com os pais embaixo de pontes, os moradores em situação de rua que precisam da boa vontade da sociedade para se alimentar, os idosos que estão cada vez mais isolados e a violência doméstica aumentada. Nas mídias, observamos o retrato de grandes filas e aglomeração de pessoas (o que não é recomendado nesta fase de pandemia) que buscam o auxílio emergencial para que possam sobreviver. Isso posto, vale lembrar que 15 famílias brasileiras têm patrimônio dez vezes maior que 14 milhões de famílias da Bolsa Família ${ }^{10}$ e que o $1 \%$ mais rico da população tem a mesma renda que os $50 \%$ mais pobres ${ }^{11}$.

Diante disso, o presente ensaio buscará refletir criticamente sobre "o que já aprendemos" em relação à educação médica no contexto atual de pandemia da Covid-19. Para isso, utilizaremos como referencial de análise reflexiva as Diretrizes Curriculares Nacionais para os Cursos de Graduação em Medicina (DCN) $)^{12}$, o Consenso Global de Responsabilidade Social das Escolas Médicas ${ }^{1}$ e a perspectiva de uma formação profissional em saúde para o século XXI'13.

\section{COMO FORMAR PROFISSIONAIS PARA O SÉCULO XXI?}

Em 2010, a revista Lancet publicou um manuscrito intitulado "Health professionals for a new century: transforming education to strengthen health systems in an interdependent world" (Profissionais de saúde para um novo século: transformando a educação para o fortalecimento dos sistemas de saúde em um mundo interdependente Tradução dos autores) ${ }^{13}$, o qual realiza uma análise histórica e socioepidemiológica sobre a formação em saúde, a fim de refletir sobre as características necessárias para uma formação profissional voltada para o século XXI. Para isso, elaborou-se uma comissão com 20 especialistas internacionais sobre o tema, que compartilharam visões e estratégias a fim de que pudessem problematizar a educação para profissionais de saúde, numa conexão entre educação e sistemas de saúde.

Há um destaque para a disparidade entre número de escolas médicas e carga de doença, o que explicita as contradições dos processos formativos em relação à responsabilidade social. É importante ressaltar que, segundo Frenk et al. ${ }^{13}$, esse problema é sistêmico, ou seja, está relacionado à falta de correspondência às reais necessidades dos(as) usuários dos sistemas de saúde; a um trabalho em equipe incipiente; à persistência das iniquidades de gênero 
e raça entre os(as) profissionais de saúde; ao enfoque educacional técnico com limitada articulação com o contexto de saúde-doença; à predominância da formação hospitalar em detrimento da atenção primária; e à falta de lideranças para melhorar a qualidade do sistema de saúde.

De acordo com um dos achados do trabalho de Frenk et al. ${ }^{13}$, há, em todo o mundo, mais de três mil instituições de formação de profissionais de saúde, as quais preparam aproximadamente um milhão desses profissionais por ano. Entretanto, há uma disparidade, marcada pela má distribuição entre os países e dentro deles. Por exemplo, quatro países (China, Índia, Brasil e Estados Unidos) têm mais de 150 escolas médicas cada um, e 36 países não apresentam sequer uma escola médica.

Ademais, a pesquisa, numa análise retrospectiva a partir do século XX, apontou que três gerações de reforma educacional caracterizaram o século passado. A primeira agregou as bases científicas nos currículos a partir do relatório Flexner. Posteriormente, no meio do século, inicialmente no Canadá, houve a inovação educacional baseada em problemas - aprendizagem baseada em problemas $(A B P)$. E a terceira geração, a qual corresponde à atual, necessita adaptar o núcleo de competências profissionais para contextos específicos, mobilizando conhecimentos, raciocínio crítico e conduta ética para a participação no sistema de saúde do indivíduo e da população ${ }^{13}$.

Nesse sentido, os autores apontam para a necessidade de repensar os currículos, para que os processos de ensinoaprendizagem estejam atrelados aos sistemas de saúde e com as reais necessidades de saúde ${ }^{12}$. Dessa forma, o currículo por si só não é o ponto de partida para se pensar a formação dos(as) futuros profissionais de saúde, mas a perspectiva de responsabilidade social adotada pela escola médica é aquela que direcionará os processos de planejamento e organização curricular. Assim, analisar as questões epidemiológicas, reconhecendo e problematizando os contextos socioculturais e a articulação com os sistemas de saúde, é um caminho possível para uma formação voltada para o século XXI em que a equidade em saúde deve ser promovida ${ }^{1,12,13}$.

Diante disso e dos 20 anos vividos em um novo século, questionamo-nos:

- Como estamos formando profissionais para o século XXI?

- Estamos reproduzindo padrões tecnicistas com uma "novaroupagem" oupromovendoaresponsabilidade social ao longo do processo formativo?

- Estamos utilizando metodologias ativas, como a ABP, com um enfoque técnico ou ampliando a análise a partir da perspectiva da determinação social do processo saúde-doença, proposta pelas $\mathrm{DCN}^{12}$ ?

- Ou seja, há, nos nossos contextos educacionais, a inserção transversal de situações reais ou simuladas com pessoas negras, lésbicas, gays, bissexuais, travestis, transexuais, indígenas, pessoas em situação de rua, pessoas privadas de liberdade, refugiados(as), entre tantos(as) outros(as) marginalizados(as) e excluídos(as) socialmente ou o nosso enfoque está predominantemente relacionado aos processos fisiopatológicos?

Neste momento, não nos propomos a trazer uma resposta, mas fomentar o debate para que, a partir dele, possamos pensar juntos(as), com os(as) vários(as) educandos(as), educadores(as), nos mais diversos contextos de nossas escolas e sistemas de saúde, quais são os caminhos possíveis para ampliarmos nossa formação e realmente pensarmos em processos formativos voltados para a promoção da equidade no século XXI. Assim, um possível caminho para encontrar algumas respostas para as questões elencadas seja observar, refletir, problematizar como temos vivenciado a pandemia da Covid-19 e como ela explicita as necessidades de aprimoramento dos processos de ensino e cuidado em saúde.

\section{O QUE APRENDEMOS NESSE CONTEXTO DE PANDEMIA DA COVID-19?}

Desde os primeiros casos da Covid-19 relatados na China, no final de 2019, e em fevereiro de 2020 no Brasi ${ }^{14}$, procuramos compreender o contexto e propor soluções para auxiliar o ensino e o cuidado em saúde. Com o passar do tempo e com as experiências de lugares que vivenciaram anteriormente ao Brasil a pandemia, fomos pensando e realizando estratégias de contenção e mitigação da pandemia da Covid-19, para posteriormente articular ações de supressão e recuperação ${ }^{14}$. Destaca-se que, nesse momento de debate e busca por ações de enfrentamento da pandemia, pôdese observar um dos elementos apontados por Frenk et al. ${ }^{13}$ para a formação voltada para o século XXI: a necessidade do diálogo entre educandos(as), educadores(as), comunidade e gestores(as) para a integração das escolas médicas com as reais necessidades de saúde e com os sistemas de saúde.

Dessa forma, o debate da formação orientada pelo e para o Sistema Único de Saúde (SUS) se torna mais evidente em nosso contexto nacional. O SUS é um dos maiores cenários de ensino-aprendizagem e de cuidado em saúde do mundo, e a pandemia da Covid-19 tem nos mostrado a relevância de fortalecermos o sistema de saúde único, universal e equânime ${ }^{14}$. Nesse sentido, é importante ressaltar que a vivência de cuidado no SUS não é igual para todas as pessoas, ainda mais em um contexto de pandemia e de grandes 
iniquidades sociais em saúde ${ }^{15}$. Estas são compreendidas como todas aquelas situações que poderiam ser modificadas e não o são e por isso perpetuam sistematicamente desvantagens para alguns(as) de se manter com saúde ${ }^{16}$. Por isso, o lema "lave as suas mãos" como uma estratégia de mitigação da propagação viral não se aplica a todas as pessoas, pois há aqueles(as) que não têm acesso à água e nem mesmo ao sabão/sabonete, por exemplo. Ou seja, este momento de pandemia explicitou as desigualdades e iniquidades no que concerne ao acesso à saúde e à promoção dela, exemplificando a importância de os processos formativos considerarem esses aspectos para que, durante as situações de cuidado, as ações dos(as) futuros(as) profissionais não reproduzam discursos e ações acríticos que reiteram e perpetuam essas situações de marginalização.

Ademais, não podemos também promover a romantização da pandemia ${ }^{17}$ e acreditar que tudo se resolve com "ficar em casa", pois nem todos têm uma casa para ficar ou "condições ideais" para trabalhar e promover a saúde em casa15. $O$ trabalho remoto tem explicitado a sobrecarga para o gênero mulher, como as cientistas mães ${ }^{18}$. Com o isolamento social, houve um aumento da violência doméstica contra mulheres, crianças e adolescentes ${ }^{19}$. Em relação à comunidade de lésbicas, gays, bissexuais, travestis, transexuais e outras identidades de gênero e orientações sexuais (LGBT+), as Nações Unidas têm apontado para o agravamento da vulnerabilidade devido à intensificação do isolamento social, da redução da rede de apoio e do aumento da violência familiar a jovens $L G B T++^{20}$. Esses e outros dados anteriormente citados exemplificam as violências estruturais ${ }^{21}$ que são perpetuadas em nossa sociedade. Por isso, um questionamento importante é:

- Será que realmente ninguém fica para trás nesta pandemia? Será que a escola médica tem se atentado a isso?

Vemos, e a literatura aponta isso, que "estamos todos [talvez] sobamesmatempestade... Masnãoestamosnomesmo barco" (p. 2)15. Quantas outras "tempestades" acometem somente determinados grupos sociais e muitas vezes sem um total auxílio daqueles(as) que estão na "calmaria"? Quais são os barcos e outras estruturas de proteção disponíveis e acessíveis à população? Sabemos por meio de várias políticas públicas $^{22-27}$ que antes mesmo da pandemia "não estávamos no mesmo barco". A epidemia da Covid-19 tem nos ensinado a observar que nós não estamos respondendo realmente ainda às necessidades da população e reduzindo de forma lenta as iniquidades em saúde.

Contudo, para isso, precisamos também olhar para aqueles(as) que compõem a escola médica. Em relação aos(às) educandos(as), temos que considerar também essas diversidades e iniquidades em saúde que vivenciam.
"Ensinamos", como educadores(as), para que os(as) discentes se protejam, que tomem os devidos cuidados diante desse contexto. Mas como ensinar a cuidar dos(as) outros(as) sem cuidar realmente daqueles(as) que ensinamos? Orientações aos(às) discentes e também aos(às) docentes e técnicos(as) administrativos(as) precisam vir acompanhadas de ações que demonstrem a responsabilidade social da instituição, inclusive com aqueles(as) que a compõem. Por exemplo, no caso de a instituição optar por manter algumas atividades acadêmicas presenciais, como os(as) estudantes, ao retornarem para os seus lares, conseguirão manter toda a infraestrutura de proteção necessária para que não haja a transmissão da Covid-19 aos(às) outros(as) familiares? Assim, mais perguntas surgem:

- Será que estamos considerando a diversidade de nossos(as) estudantes, de suas condições socioculturais e familiares neste momento de pandemia?

- Será que estamos buscando promover um ensino em tempo de pandemia ou contando somente com a "mão de obra não paga" dos(as) estudantes?

Ainda sobre os processos formativos, anteriores ou não à pandemia, há um estímulo e uma construção social para assumirmos e reproduzirmos a posição de heróis/heroínas ${ }^{17,28}$ : "Precisamos salvar vidas", "Somos aqueles(as) que salvam as pessoas dessa doença" e "Os verdadeiros heróis". E tantas outras frases e discursos que, incialmente, reiteram uma perspectiva machista de gênero por associar majoritariamente o "heroísmo" ao atributo do masculino. Ademais, essas perspectivas desconsideram o sofrimento psicoemocional dos(as) profissionais de saúde e dos(as) estudantes, pois, ao assumirmos essa postura "heroica", assumimos também essa figura de não poder sofrer, não poder reclamar, não poder desistir ${ }^{28,29}$. Entretanto, é curioso que, neste momento, a máscara muitas vezes utilizada pelos(as) personagens heroicos que compõem a nossa imagem mental hegemônica não seja mais tão útil, pois deixa desprotegida, normalmente, a boca e o nariz. Talvez essa necessidade, durante a pandemia, do uso de máscaras faciais que cubram boca e nariz seja o convite para pensarmos em uma humanidade para além de heróis/heroínas ${ }^{29}$.

É importante, assim, pensarmos e produzirmos uma formação mais humanística e que o trabalho profissional em saúde seja uma responsabilidade de equipe(s) e não de um indivíduo isoladamente ${ }^{1,13}$. Assim como as $\mathrm{DCN}^{12}$ apontam, a pandemia explicita a necessidade do trabalho multiprofissional e interprofissional para o século XXI. Esse trabalho deve ser realizado em diálogo constante com a comunidade, de modo a promover a educação em saúde que considere as diversidades e a determinação social do processo saúde-doença ${ }^{12}$. Para isso, 
a educação médica precisa promover a comunicação efetiva, afetiva e não violenta entre os(as) vários(as) sujeitos(as) envolvidos(as) no cuidado. A pandemia - e os contextos que a antecedem - nos trouxe vários exemplos dessa necessidade da comunicação e do trabalho em equipe, como o trabalho de combate às fake news (notícias/informações erradas), a epidemia de desinformação ${ }^{30}$. Talvez a educação em saúde, prevista nas $D_{C N}{ }^{12}$, e até na forma de educação popular em saúde ${ }^{31}$, possa utilizar os dispositivos móveis como aliados ${ }^{32,33}$ na busca pela promoção da equidade.

Para promover a equidade, tanto nos processos educacionais quanto nos de cuidado em saúde, a solidariedade torna-se um caminho necessário34: uma "solidariedade de fato, solidariedade de ação, solidariedade concreta para as pessoas em termos de equipamentos e dinheiros" (p. 1086)35 e políticas públicas. Ou seja, este momento da pandemia da Covid-19 nos ensinou a importância de ações solidárias concretas como recursos de efetivação da responsabilidade social. Um exemplo foi a ação de educadoras de uma universidade federal em arrecadar alimentos e produtos de higiene e limpeza para as travestis do município que, por conta do isolamento social, não podiam trabalhar ${ }^{36}$. Essa iniciativa estimulou a universidade a promover uma ação constante e ampliada para outros grupos vulnerabilizados, promovendo redes de solidariedade e comunidades de práticas ${ }^{37}$ que apontam para o aprimoramento da responsabilidade social e sua importância social, para além da produção de conhecimento propriamente dito.

Diante de tudo isso, Hall et al. ${ }^{38}$ destacam a importância de cinco elementos para que se consiga avançar durante este momento de pandemia: comunicação, inovação, colaboração, planejamento e flexibilidade. Considerar esses aspectos atrelados à diversidade, às reais necessidades de saúde da população, aos sistemas de saúde e às situações de iniquidades em saúde potencializará a promoção da equidade e a formação de profissionais do/no século XXI comprometidos(as) com a responsabilidade social.

\section{CONSIDERAÇÕES FINAIS}

Precisamos articular os processos de ensinoaprendizagem com os sistemas de saúde, com as reais necessidades de saúde da população; o ensino e o cuidado que promovam a diversidade e reduzam as iniquidades em saúde; uma formação e prática em equipe; a promoção de saúde mental e da educação em saúde; e as redes de solidariedade.

Precisamos advogar em função dos usuários do SUS e também despertar em nossos(as) estudantes o olhar para essas necessidades. Os(as) profissionais formados(as) devem ser comprometidos(as) com a responsabilidade social, e não cabe mais formar pessoas que não tenham essa competência.
As demandas sociais da atualidade também exigem competências atitudinais e relacionais dos(as) profissionais da saúde, o que precisa ser colocado em prática na formação desses(as) profissionais. Ser sensível neste mundo requer muita coragem, e os(as) profissionais de saúde devem ver além do que os olhos podem ver, e isso é muito importante.

Além do mais, acreditamos como educadora e educador que somos, que é necessário o esperançar nos processos de ensino e cuidado, como no atual momento de pandemia. A esperança deve ser uma ação concreta para que possamos transformar as realidades difíceis que temos vivenciado em realidades mais equânimes, pois "não há mudança sem sonho, como não há sonho sem esperança” (p. 172-173)34.

\section{AGRADECIMENTOS}

Agradecemos a todos/as os/as integrantes do grupo de trabalho Populações (In)Visibilizadas e Diversidades da Associação Brasileira de Educação Médica (Abem). Agradecemos também a Suelly Grosseman, Denise Herdy e Sandro Scraiber em nome da diretoria da gestão 2018-2020 da Abem que, por meio do "Painel Abem - A escola médica na epidemia da Covid-19: o que já aprendemos?”, potencializaram as reflexões deste manuscrito. Agradecemos também a todas as pessoas que de alguma forma apoiam essa luta.

\section{CONTRIBUIÇÃO DOS AUTORES}

Gustavo Antonio Raimondi e Francis Solange Vieira Tourinho foram responsáveis pela conceituação do trabalho, curadoria de dados, análise formal, investigação, metodologia e redação (rascunho original, revisão e edição).

\section{CONFLITO DE INTERESSES}

Os autores declararam não haver potenciais conflitos de interesse com relação à pesquisa, autoria e/ou publicação deste artigo.

\section{REFERÊNCIAS}

1. The Net. Global Consensus for Social Accountability of Medical Schools. Vancouver; 2010. Disponível em: http://healthsocialaccountability.sites. olt.ubc.ca/files/2011/06/11-06-07-GCSA-English-pdf-style.pdf. Acesso em: 03 ago 2020.

2. Boelen C. Global Consensus on the Social Accountability of Medical Schools. Sante Publique. 2011;23(3):247-50.

3. Patel JA, Nielsen FBH, Badiani AA, Assi S, Unadkat VA, Patel B, et al. Poverty, inequality and COVID-19: the forgotten vulnerable. Public Health. 2020;183:110-1.

4. Andersen M. Early evidence on social distancing in response to COVID-19 in the United States. 2020. Disponível em: https://papers.ssrn.com/sol3/ papers.cfm?abstract_id=3569368. Acesso em: 03 ago 2020.

5. Instituto Brasileiro de Geografia e Estatística. Coordenação de população, indicadores sociais, síntese de indicadores sociais: uma análise das condições de vida da população brasileira: 2019. Brasília: IBGE; 2019. 
6. Matos CC, Tourinho FS. Saúde da população negra: como nascem, vivem e morrem os indivíduos pretos e pardos em Florianópolis (SC). Rev Bras Med Fam Comunidade. 2018;13(40):1-3.

7. Santos DD, Menezes MO, Andreucci CB, Nakamura-Pereira M, Knobel R, Katz L, et al. Disproportionate impact of COVID-19 among pregnant and postpartum Black Women in Brazil through structural racism lens. Clin Infect Dis. 2020 Jul 28. Disponível em: https://academic.oup.com/cid/ advance-article/doi/10.1093/cid/ciaa1066/5877027. Acesso em: 03 ago 2020.

8. Lancet T. Redefining vulnerability in the era of COVID-19. Lancet. 2020;395(10230):1089.

9. Smith JA, Judd J. COVID-19: vulnerability and the power of privilege in a pandemic. Health Promot J Austr. 2020 Apr;31(2):158-60.

10. A lista dos bilionários brasileiros. Forbes out. 2019; ed esp, ano VII, n. 71.

11. Barros RP, Foguel MN, Ulyssea GO. Desigualdade de renda no Brasil: uma análise da queda recente. Brasília: Ipea, 2006.

12. Brasil. Resolução $n^{\circ} 3$, de 20 de junho de 2014. Institui Diretrizes Curriculares Nacionais do Curso de Graduação em Medicina e dá outras providências. Brasília; 2014.

13. Frenk J, Chen L, Bhutta ZA, Cohen J, Crisp N, Evans T, et al. Health professionals for a new century: transforming education to strengthen health systems in an interdependent world. Lancet. 2010;376(9756):1923-58.

14. Werneck GL, Carvalho MS. A pandemia de COVID-19 no Brasil: crônica de uma crise sanitária anunciada. Cad Saude Publica, 2020;36(5):e00068820.

15. Minayo MCS, Freire NP. Pandemia exacerba desigualdades na saúde. Cien Saude Colet. 2020; 25(9):3555-6. Disponível em: http:// www.cienciaesaudecoletiva.com.br/artigos/pandemia-exacerbadesigualdades-na-saude/17579?id=17579.

16. Barata RB. Como e por que as desigualdades sociais fazem mal à saúde. Rio de Janeiro: Fiocruz; 2009.

17. Xavier A. O grande desafio da pandemia é emocional, ético e social. RADIS. 2020;(213):32-3. Disponível em: https://www.arca.fiocruz.br/ bitstream/icict/41754/2/GrandeDesafio.pdf. Acesso em: 03 ago 2020.

18. Staniscuaski F, Reichert F, Werneck FP, Oliveira L de, Mello-Carpes PM, Soletti RC, et al. Impact of COVID-19 on academic mothers. Science. 2020;368(6492):724.

19. Marques ES, Moraes CL, Hasselmann MH, Deslandes SF, Reichenheim ME. A violência contra mulheres, crianças e adolescentes em tempos de pandemia pela COVID-19: panorama, motivações e formas de enfrentamento. Cad Saude Publica. 2020;36:e00074420.

20. United Nations Human Rights - Office of the Hight Commissioner. COVID-19 and the human rights of LGBTI people. 2020 [acesso em 10 ago 2020]. Disponível em: https://www.ohchr.org/Documents/Issues/LGBT/ LGBTIpeople.pdf.

21. Cruz Neto $O$, Moreira MR. A concretização de políticas públicas em direção à prevenção da violência estrutural. Cien Saude Colet. 1999;4(1):33-52.

22. Brasil. Política Nacional de Atenção à Saúde dos Povos Indígenas. Brasília: Funasa; 2002.

23. Brasil. Política Nacional de Atenção Integral à Saúde da Mulher: princípios e diretrizes. Brasília: Ministério da Saúde; 2004.
24. Brasil. Política Nacional para Inclusão da População em Situação de Rua. Brasília: Ministério da Saúde; 2008.

25. Brasil. Política Nacional de Atenção Integral à Saúde da População Negra: uma política do SUS. Brasília: Ministério da Saúde; 2013.

26. Brasil. Política Nacional de Saúde Integral de Lésbicas, Gays, Bissexuais, Travestis e Transexuais. Brasília: Ministério da Saúde; 2013.

27. Brasil. Políticas de Promoção da Equidade em Saúde. Brasília: Ministério da Saúde; 2013.

28. Ornell F, Halpern SC, Kessler FHP, Narvaez JCM. The impact of the COVID-19 pandemic on the mental health of healthcare professionals. Cad Saude Publica. 2020; 36(4):e00063520.

29. Raimondi GA. Between Applauses and loneliness, heroes/warriors and fear: thoughts of a medical professor during the COVID-19 pandemic. Qualitative Inquiry. 2020 Jul 13;107780042094105. doi: 10.1177/1077800420941051.

30. Sousa Júnior JH, Raasch M, Soares JC, de Sousa LV. Da desinformação ao caos: uma análise das fake news frente à pandemia do coronavírus (COVID-19) no Brasil. Cadernos de Prospecção. 2020;13 (2 Covid-19):331-46.

31. Brasil. Portaria $n^{\circ} 2.761$, de 19 de novembro de 2013. Institui a Política Nacional de Educação Popular em Saúde no Âmbito do Sistema Único de Saúde (PNEPSSUS). Brasília; 2013. Disponível em: bvms.saúde.gov.br/ bvs/saudelegis. Acesso em: 03 ago 2020.

32. Paulino DB, Martins CCA, Raimondi GA, Hattori WT. WhatsApp ${ }^{\circledR}$ como recurso para a educação em saúde: contextualizando teoria e prática em um novo cenário de ensino-aprendizagem. Rev Bras Educ Med. 2018;42(1):171-80.

33. Bernardes VP, Dias LF, Pereira MA, Fernandes ME, Raimondi GA, Paulino DB. Facebook ${ }^{\circledR}$ como ferramenta pedagógica em saúde coletiva: integrando formação médica e educação em saúde. Rev Bras Educ Med. 2019;43(1):652-61.

34. Freire P. Pedagogia da esperança: um reencontro com a pedagogia do oprimido. 21a ed. São Paulo: Paz e Terra; 2014.

35. Shamasunder S, Holmes SM, Goronga T, Carrasco H, Katz E, Frankfurter $R$, et al. COVID-19 reveals weak health systems by design: why we must re-make global health in this historic moment. Glob Public Health. 2020; 15(7):1083-9. doi: 10.1080/17441692.2020.1760915.

36. Ribeiro L. Professoras da UFU criam campanha de arrecadação para transexuais e travestis. Entrevista - Comunica UFU. 2020. Disponível em: http://www.dirco.ufu.br/noticia/2020/04/professoras-da-ufu-criam-campanhade-arrecadacao-para-transexuais-e-travestis. Acesso em: 03 ago 2020.

37. Wenger-Trayner E, Wenger-Trayner B. Introduction to communities of practice: a brief overview of the concept and its uses. 2015. Disponível em: https://wenger-trayner.com/wp-content/uploads/2015/04/07-Briefintroduction-to-communities-of-practice.pdf. Acesso em: 03 ago 2020.

38. Hall AK, Nousiainen MT, Campisi P, Dagnone JD, Frank JR, Kroeker KI, et al. Training disrupted: practical tips for supporting competencybased medical education during the COVID-19 pandemic. Med Teach. 2020:42(7):756-61. 